\title{
Questioning the status of aberrant speech patterns as psychiatric symptoms
}

\author{
Eric J. Tan and Susan L. Rossell
}

\section{Summary}

Speech disturbances manifest in various psychiatric conditions and demonstrate temporal variability in relation to acute and stable symptom periods. They can be externally assessed, which facilitates their potential use as an objective marker of illness stage. Continued research will have positive implications for diagnostics and long-term management in clinical settings.

\section{Keywords}

Nosology; psychotic disorders; schizophrenia; formal thought disorder; speech.

\section{Copyright and usage}

(c) The Authors 2020
Eric J. Tan (pictured) is an NHMRC Early Career Research Fellow at Swinburne University of Technology, Melbourne, Australia, with interests in psychiatric speech, language and cognitive phenomena. Susan L. Rossell is an NHMRC Senior Research Fellow at Swinburne University of Technology, Melbourne, Australia, with expertise in psychiatric speech, symptoms, cognition and neurobiology.

Speech disturbances have been considered a core component of various psychiatric conditions over the past century, most notably schizophrenia. A key schizophrenia diagnostic criterion, these speech abnormalities have traditionally been assessed via clinical interviews using standardised rating scales - of which a number are available - with the vast majority of work, to date, focusing on the structure or form of speech. Recognised speech disturbances range from nonsensical sentences (incoherence), tangential replies (tangentiality) and losing the aim of the response (loss of goal), to monosyllabic conversation (poverty of speech) and rapid verbose speech (pressure of speech).

Traditionally referred to as formal thought disorder (FTD), these aberrant speech patterns were once considered pathognomonic of schizophrenia, but have now also been observed in other psychiatric conditions, such as bipolar disorder. ${ }^{1}$ The non-specificity of speech disturbances to a single psychiatric condition is perhaps contradictorily juxtaposed with schizophrenia still having FTD as a diagnostic symptom. Although poverty of speech and pressure of speech appear to be more closely associated with schizophrenia and bipolar disorder, respectively, ${ }^{1}$ the majority of FTD manifestations are not diagnosis-specific. This suggests that a reassessment of the ontology of FTD, and of speech disturbances in general, is both appropriate and required.

\section{Is FTD a schizophrenia symptom?}

The status of FTD as a schizophrenia symptom is challenged not only by its non-diagnostic specificity, but also by evidence that FTD is absent in up to $73 \%$ of all patients with schizophrenia, depending on the assessed cohort. ${ }^{2}$ It additionally differs in severity among those who have it, which reflects its multidimensional presentation (e.g. verbose versus disorganisation-type speech). ${ }^{3}$ Another key feature of FTD is its temporal variability, in that it waxes and wanes within patients across time in relation to stage of illness, with highest severity in the acute stage and significantly reduced severity or absence during stable periods. ${ }^{2}$ This closely mirrors other symptom fluctuations in schizophrenia, and is similar to bipolar disorder-related speech disturbances. ${ }^{1}$ These features collectively reduce the diagnostic utility of FTD for schizophrenia and suggest that although FTD is not a schizophrenia-specific symptom, it might instead be a suitable marker for psychosis in general.

A symptom is medically defined as an experience that is only recognisable by the person and has to be self-reported, such as abdominal pain. A sign is a phenomenon that can be externally observed by another individual, such as a fever. Notably, signs can also be self-reported, thus not precluding a sign from also being a symptom, but the same cannot be said of the converse. In psychiatry, speech disturbances/FTD hold the unique position of being psychotic phenomena that can be objectively assessed by others, unlike delusions and hallucinations that rely exclusively on patient selfreport. Patients are also often unaware of their incongruent or unusual speech patterns, which reduces the reliability of selfreported FTD measures (self-reported FTD scales may be tapping into slightly different constructs to the traditional speech-centric conceptualisations of FTD, and as a relatively recent development in the field, diagnostic criteria still call for clinically assessed FTD as the standard measure). Accordingly, this also suggests that FTD is less of a symptom and more of a sign of the underlying disorder.

\section{What constitutes a psychiatric marker?}

A marker can be biologically defined as a characteristic feature that indicates something is happening or likely to happen. In relation to psychosis, a marker would thus be any state-based feature that is present during psychotic episodes, and fluctuates relative to symptom severity or the imminent onset of psychosis. In line with this, the utility of FTD as a marker for psychosis can be supported by four key points. First, its aforementioned presence in a variety of psychiatric conditions; second, its temporal variability across the illness course between acute and stable stages; third, FTD is increasingly recognised as having more neurocognitive origins, ${ }^{4}$ with aetiological independence from other features of psychosis supporting the argument that it is actually a marker; and fourth, FTD can be externally assessed, which reflects a major advantage of speech assessment in general over existing clinical interviews in psychiatry, where a speedy and objective tool is still elusive. However, current assessments of FTD are admittedly still based on clinical rating scales that are not ideal because of issues of subjectivity and lengthy assessment durations. This highlights the need to harness objective speech assessment protocols and move beyond FTD, to further the development of clinical markers in psychiatry. 


\section{Speech disturbances as a psychiatric marker}

There are a growing number of different techniques, including machine learning and digital delivery, that are building the case for increased speech assessment in psychiatry. Apart from the more conventional conceptualisations of aberrant speech, such as FTD and communication disturbances, other aspects of speech are increasingly being examined, such as pitch, speaking rate and number of pauses. These measures can be more objectively obtained compared to conventional psychiatric speech assessment methods, such as FTD scales. This increasing capacity supports future development and establishment of assessing speech disturbances as a marker of psychosis. Consequential translation of this would permit increased objectivity, accuracy and efficiency of diagnosis in research scenarios and in the identification and management of symptoms in clinical settings.

As noted, not all patients with psychosis demonstrate FTD. However, this should be interpreted in light of previous study methodologies (and classification thresholds of FTD versus non-FTD), and a lack of focus on temporality. A thorough speech assessment, by objective acoustic voice-based measures (e.g. pitch), will address existing limitations of FTD assessment, including subclinical threshold features and between-patient heterogeneity. Further ascertaining how these speech abnormalities vary according to illness trajectory and are validated with existing diagnostic techniques will be of critical importance. The latter point is critical given the recognised issues with diagnostic rigour in psychiatry at present, and parallels with the multiple stages in the process of establishing biomarkers (i.e. confirmation, validation, replication and ratifying specificity and sensitivity). A plethora of work lies ahead for this endeavour.

The examination of speech disturbances, as a marker for psychosis, is an opportunity to adopt a Gestalt-like approach toward understanding the relationship between psychotic symptoms and speech. This holistic approach will hopefully circumvent the cattywampus present-day conceptualisations of psychiatric speech disturbances and how they should be assessed. Such work will be critical to building support for speech disturbances as a marker for psychosis as a first step. Speech assessment should subsequently be investigated in relation to negative symptoms, which would facilitate utility in distinguishing between affective and non-affective psychoses, as well as in other conditions with recognised speech abnormalities, such as delirium, dementia and traumatic brain injury.

\section{A broader approach beyond diagnostics}

Poor diagnostic utility of FTD is contrasted by evidence for good prognostic utility and relationship to quality of life. ${ }^{2}$
Consequently, the study of FTD should continue to be advocated and facilitate the definitive characterisation of its underpinnings, and its relationship to psychotic symptoms. Newer, and possibly independent to FTD, measures of speech disturbances (e.g. pitch) also need to be investigated for their diagnostic and prognostic utility. The vast majority of speech studies have focused on schizophrenia spectrum and bipolar disorders. Strengthening the case for speech disturbances as markers of psychosis is contingent on continued research in other psychotic disorders as well.

Eric J. Tan (D), Centre for Mental Health, Swinburne University of Technology, Australia; Susan L. Rossell, Centre for Mental Health, Swinburne University of Technology, Australia

Correspondence: Eric J. Tan. Email: erictan@swin.edu.au

First received 12 Sep 2019, final revision 18 Feb 2020, accepted 27 Feb 2020

\section{Author contributions}

Both E.J.T. and S.L.R. conceptualized the manuscript. E.J.T. wrote the first draft and S.L.R. provided feedback and review toward the final manuscript. Both E.J.T. and S.L.R. approve of the version to be published.

\section{Funding}

The authors received no financial support for the research, authorship and/or publication of this article. E.J.T. and S.L.R. are in receipt of Early Career (GNT1142424) and Senior Research (GNT1060664) fellowships, respectively, from the National Health and Medical Research Council of Australia.

\section{Declaration of interest}

\section{None.}

\section{References}

1 Yalincetin B, Bora E, Binbay $\mathrm{T}$, Ulas H, Akdede BB, Alptekin K. Formal thought disorder in schizophrenia and bipolar disorder: a systematic review and metaanalysis. Schizophr Res 2017; 185: 2-8.

2 Roche E, Creed L, MacMahon D, Brennan D, Clarke M. The epidemiology and associated phenomenology of formal thought disorder: a systematic review. Schizophr Bull 2015; 41(4): 951-62

3 Tan EJ, Rossell SL. On the dimensionality of formal thought disorder. Schizophr Res 2019; 210: 311-2.

4 Tan EJ, Rossell SL. On the nosology of formal thought disorder. Aust N Z J Psychiatry 2015; 49(8): 758-9.

5 Rapcan V, D'Arcy S, Yeap S, Afzal N, Thakore J, Reilly RB. Acoustic and temporal analysis of speech: a potential biomarker for schizophrenia. Med Eng Phys 2010; 32(9): 1074-9. 